\title{
Proteomic discovery and verification of serum amyloid $A$ as a predictor marker of patients at risk of post-stroke infection: a pilot study
}

\author{
L. Azurmendi ${ }^{1 \dagger}$, V. Lapierre-Fetaud ${ }^{1 \dagger}$, J. Schneider ${ }^{2}$, J. Montaner $^{3}$, M. Katan ${ }^{2 \dagger}$ and Jean-Charles Sanchez ${ }^{{ }^{*+}}$
}

\begin{abstract}
Background: Post-stroke infections occur in $20-36 \%$ of stroke patients and are associated with high morbidity and mortality rates. Early identification of patients at risk of developing an infection could improve care via an earlier treatment leading to a better outcome. We used proteomic tools in order to discover biomarkers able to stratify patients at risk of post-stroke infection.

Methods: The post hoc analysis of a prospective cohort study including 40 ischemic stroke patients included 21 infected and 19 non-infected participants. A quantitative, isobaric labeling, proteomic strategy was applied to the plasma samples of 5 infected and 5 non-infected patients in order to highlight any significantly modulated proteins. A parallel reaction monitoring (PRM) assay was applied to 20 additional patients (10 infected and 10 non-infected) to verify discovery results. The most promising protein was pre-validated using an ELISA immunoassay on 40 patients and at different time points after stroke onset.

Results: Tandem mass analysis identified 266 proteins, of which only serum amyloid A (SAA1/2) was significantly $(p=0.007$ ) regulated between the two groups of patients. This acute-phase protein appeared to be 2.2 times more abundant in infected patients than in non-infected ones. These results were verified and validated using PRM and ELISA immunoassays, which showed that infected patients had significantly higher concentrations of SAA1/2 than non-infected patients at hospital admission, but also at 1, 3, and 5 days after admission.

Conclusions: The present study demonstrated that SAA1/2 is a promising predictor, at hospital admission, of stroke patients at risk of developing an infection. Further large, multicenter validation studies are needed to confirm these results. If confirmed, SAA1/2 concentrations could be used to identify the patients most at risk of post-stroke infections and therefore implement treatments more rapidly, thus reducing mortality.
\end{abstract}

\section{Background}

Stroke is a serious medical condition produced by brain cell death. It occurs when there is a lack of blood flow to the brain ( $80 \%$ of cases) or a hemorrhage affecting the brain or its surroundings (20\%). Every year, around

\footnotetext{
${ }^{*}$ Correspondence: jean-charles.sanchez@unige.ch

${ }^{\dagger}$ L. Azurmendi, V. Lapierre-Fetaud, M. Katan and Jean-Charles Sanchez contributed equally to this work

${ }^{1}$ Translational Biomarker Group, Department of Human Protein Sciences, University of Geneva, Rue Michel Servet 1, 1211 Geneve 4, Switzerland Full list of author information is available at the end of the article
}

15 million people will suffer a stroke, leading to 6 million deaths and 5 million disabled patients [1-3]. Around 40\% of patients die within the first weeks following the stroke $[4,5]$. Non-modifiable factors, such as the severity of the stroke or the age of the patient, are highly correlated with mortality [6, 7]. However, one-third of deaths result from potentially preventable stroke-associated complications. Nosocomial infection, particularly bacterial pneumonia, is the most common complication after stroke, with an incidence of 5-22\% [8-10]. Despite the intensive care given to these patients, infection rates remain elevated and are 
associated with bad functional outcome and mortality [11]. The high incidence of infection is likely to be a result of an impaired immune function. The patient's reduced ability combat bacteria is a consequence of the initial brain damage $[12,13]$. Therefore, the early identification of patients who might be prone to developing an infection after stroke is a necessary step towards better hospital management, more rapid implementation of treatments, and improved long-term patient outcomes [13, 14].

In clinical practice, the diagnosis of post-stroke infection is a challenging one as there has been no satisfactory concordance between different studies. The most widely studied markers of post-stroke infection-procalcitonin (PCT), C-reactive protein (CRP), and white blood cells (WBC) - have only shown moderate predictive value, and their levels do not increase early enough to be of help before the infection is clinically apparent $[15,16]$. Clinical signs such as older age, fever, severe stroke, or dysphagia, among others, have been linked to post-stroke associated pneumonia [17]. Nevertheless, they are not specific enough to act as individual markers. Using a combination of these markers with clinical scales such as the A2DS2, AIS-APS, and ISAN [17-19] have not been applied routinely in clinical practice. The gold standard for diagnosing an infection is the result from a bacterial culture, yet this may take 2 days. All of these reasons can lead to antibiotic treatment being started too late, with the unfortunate associated consequences. There is thus evidence of a need for a reliable early biomarker [20].

The present study aimed to use proteomic approaches to find a biomarker that could be tested for at hospital admission in order to identify patients at risk of developing a post-stroke infection. To do this, we investigated the plasma proteomes of infected and non-infected patients, using isobaric labeling methods. After selecting SAA1/2 as the most promising protein, parallel reaction monitoring (PRM) and the enzyme-linked immunosorbent assays (ELISA) confirmed its ability to predict which patients were at risk of infection after a stroke.

\section{Methods}

\section{Study design and setting}

We performed a post hoc analysis of a prospective cohort study which included 40 ischemic stroke patients (ClinicalTrials.gov.NCT00390962) who had been hospitalized consecutively at the University Hospital of Basel (Switzerland) between November 2006 and November 2007. The study protocol was conducted according to the principles expressed in the Declaration of Helsinki and with the approval of the local ethics committee. Before enrolment, informed consent was obtained from patients, their relatives, or their legal guardians.

\section{Clinical protocol}

Comprehensive information on the assessment of the study participants' demographic and vascular risk factors has been published previously [15]. Briefly, ischemic stroke was defined according to the World Health Organization criteria [21]. A detailed history was obtained for vascular risk factors, vital signs, and relevant comorbidities as assessed using the Charlson Comorbidity Index (CCI) medication taken prior to the stroke. Neurological deficits were estimated using the National Institutes of Health Stroke Scale (NIHSS). Patients underwent the following standardized diagnostic workup: brain computer tomography $(\mathrm{CT})$ and/or magnetic resonance imaging, long-term electrocardiography, echocardiography, and neurosonographic imaging of the extracranial and intracranial arteries. Stroke etiology was determined according to the TOAST (Trial of Org 10172 in Acute Stroke Treatment) classification criteria, which distinguish largeartery arteriosclerosis, cardio embolism, small-artery occlusion, other etiologies, and undetermined etiologies [22].

\section{Definition of stroke-associated Infections}

Stroke-associated infection (SAI) was defined as any infection occurring within the first 5 days after hospital admission [13]. Infections were diagnosed according to the U.S. Centers for Disease Control and Prevention (CDC) criteria [15]. We distinguished between pneumonia, urinary tract infection (UTI), and other infections (OI). Pneumonia was diagnosed when at least one symptom from each of the two following symptom groups was present: (1) abnormal respiratory examination, pulmonary infiltrates in chest X-rays; (2) productive cough with purulent sputum, positive microbiological cultures from the lower respiratory tract or blood cultures.

Diagnosis of a UTI required two of the following criteria to be met: fever $\left(\geq 38.0{ }^{\circ} \mathrm{C}\right)$, urine sample positive for nitrite, leukocyturia $(\geq 40 / \mu \mathrm{L})$, or significant bacteriuria ( $\geq 10^{4} / \mathrm{mL}$ of an uropathogen). OI were diagnosed if white blood cell count was $\geq 11,000 / \mathrm{mL}$ and CRP was $\geq 10 \mathrm{mg} / \mathrm{L}$ or temperature was $\geq 38.0^{\circ} \mathrm{C}$ and an infectious manifestation was present. The treating physician made the diagnosis of pneumonia during hospitalization. This was then validated post hoc using charts.

The time point of diagnosis was taken to be the beginning of clinical symptoms which led to the diagnostic workup and resulted in the diagnosis of infection. In order to exclude any acute infections that had preceded the stroke, patients with an admission temperature $>38^{\circ} \mathrm{C}$, reporting an infection lasting up to 3 days before the onset of stroke, or who required mechanical intubation were not included in the study. 


\section{Blood sample collection}

Blood samples were collected from venous blood puncture within the first $72 \mathrm{~h}$ following symptom onset and then 1,3 , and 5 days after admission. Blood was centrifuged for $30 \mathrm{~min}$ at $3000 \times g$, collected in EDTA, tubes and stored at $-80^{\circ} \mathrm{C}$.

\section{Proteomic study}

\section{Quantitative proteomic analysis: TMT}

Quantitative proteomic analyses were performed on five infected and five non-infected patients at hospital admission. The aim was to identify significantly regulated proteins between the two groups in order to find a promising infection marker.

Reduction, alkylation, digestion, and TMT labeling The quantitative proteomic experiment used $1 \mu \mathrm{L}$ of each plasma sample. These amounts were dried and reconstituted in $16.6 \mu \mathrm{L}$ of $6 \mathrm{M}$ urea in tetraethylammonium bromide (TEAB) $0.1 \mathrm{M}$. The proteins were reduced by adding $1 \mu \mathrm{L}$ of $50 \mathrm{mM}$ tris-(2-carboxyethyl) phosphine hydrochloride (TCEP) to each sample, and they then reacted for $1 \mathrm{~h}$ at $37^{\circ} \mathrm{C}$. After the sample had cooled to room temperature, the alkylation step required mixing the solution with $1 \mu \mathrm{L}$ of iodoacetamide $400 \mathrm{mM}$ and storage at room temperature for $30 \mathrm{~min}$. Sixty seven $\mu \mathrm{L}$ of TEAB $0.1 \mathrm{M}$ were added to reduce the concentration of urea to $<2 \mathrm{M}$. The digestion was carried out overnight at $37^{\circ} \mathrm{C}$ using $1 \mu \mathrm{g}$ of trypsin for each $20 \mu \mathrm{g}$ of protein. The protocol is detailed by Dayon et al. [23, 24].

Subsequently, each digested plasma sample was labeled with one of the 10 TMT reagents (Thermo Fisher Scientific, Waltham, USA). Infected patients' samples were labeled with TMTs 127n, 128n, 129n, 130n, and 131n. Non-infected patients' samples were labeled with TMTs $126,127 \mathrm{c}, 128 \mathrm{c}, 129 \mathrm{c}$, and 130c. To calculate experimental error, $1 \mu \mathrm{g}$ of $\beta$-lactoglobulin was spiked in each sample. All the samples were pooled, desalted using a C18 Macro SpinColumn, and dried in a speed-vacuum.

Off-gel electrophoresis (OGE) OGE separation was carried out using an Agilent 3100 Off-Gel fractionator, as per the manufacturer's instructions. Previously dried samples were reconstituted using the OGE solution and then focused using an immobilized pH gradient (IPG) dry strip (13 cm, pH 3-10) [23]. After OGE, samples were desalted using a C18 Micro SpinColumn, dried in the speed-vacuum, and stored at $-20^{\circ} \mathrm{C}$ until analysis.

$L C-M S / M S$ A Q-Exactive Plus mass spectrometer (ThermoFisher, San Jose, CA), coupled with nanoflow high-pressure liquid chromatography (HPLC), was used to analyze the OGE fractions, as previously described [25].
Briefly, peptides reconstituted using 5\% CAN, 0.1\% FA, were trapped in a $5 \mu \mathrm{m} 200 \AA$ Magic C18 AQ (Michrom) $0.1 \times 20 \mathrm{~mm}$ pre-column and separated in a $5 \mu \mathrm{m} 100 \AA$ Magic C18 AQ (Michrom) $0.75 \times 150 \mathrm{~mm}$ column with a gravity-pulled emitter. Both columns were made inhouse. The analytical separation ran for 65 min using a gradient of $\mathrm{H}_{2} \mathrm{O} / \mathrm{FA} 99.9 / 0.1 \%$ (solvent $\mathrm{A}$ ) and $\mathrm{CH}_{3} \mathrm{CN} /$ FA $99.9 / 0.1 \%$ (solvent $\mathrm{B}$ ). The gradient ran at a flow rate of $220 \mathrm{~nL} / \mathrm{min}$ as follows: $0-1 \mathrm{~min} 95 \% \mathrm{~A}$ and $5 \% \mathrm{~B}$, then to $65 \% \mathrm{~A}$ and $35 \% \mathrm{~B}$ at $55 \mathrm{~min}$, and $20 \% \mathrm{~A}$ and $80 \% \mathrm{~B}$ at $65 \mathrm{~min}$. For the MS survey scans, OT resolution was set to 60,000 and the ion population was set to $5 \times 105$ with an $\mathrm{m} / \mathrm{z}$ window from 400 to 2000 . A maximum of three precursors were selected for both collision-induced dissociation (CID) in the LTQ and higher-energy collisional dissociation (HCD) with analysis in the OT. For MS/MS in the LTQ, the ion population was set to $7 \times 10^{3}$ (isolation width of $2 \mathrm{~m} / \mathrm{z}$ ), whereas for MS/MS detection in the OT, it was set to $2 \times 10^{5}$ (isolation width of $2.5 \mathrm{~m} / \mathrm{z}$ ), with a resolution of 7500 , a first mass at $\mathrm{m} / \mathrm{z}=100$, and a maximum injection time of $750 \mathrm{~ms}$. The normalized collisional energies were set to $35 \%$ for CID and 60\% for HCD.

Protein identification MS data were processed using EasyProtConv. Peak lists were obtained using the 12 OGE fractions and the combination of HCD-CID raw data peak lists were generated. Afterwards, these data were submitted to an EasyProt software platform (version 2.3, build 718) that uses Phenyx software (GeneBio, Geneva, Switzerland) for protein identification. The protein search was made using the Uniprot/Swiss-Prot database (2014-10, 669903) [26], applying the following search criteria: Homo sapiens taxonomy, oxidized methionine (as the variable modification), and cysteine carbamethylation, $\mathrm{TMT}^{10}$ lysine, and $\mathrm{TMT}^{10}$ amino-terminus (as the fixed modifications). Trypsin was selected as the proteolytic enzyme, allowing one missed cleavage. Parent-ion tolerance was set to $10 \mathrm{ppm}$ and the accuracy of fragment ions to $0.6 \mathrm{Da}$. Only proteins with a less than $1 \%$ false discovery rate (FDR) and at least two different unique peptides were selected for further analysis [27]. A minimum peptide length of 6 amino acids was used.

Protein quantification used the Isobar R package [28]. The manufacturer's isotopic distribution data was used to correct the isotopic impurities of $\mathrm{TMT}^{10}$ reporter-ion intensities. The equal median intensity method was used to normalize the reporter intensities. Peptides which did not present reporter intensities were not quantified. The infection/no infection ratio was calculated for each peptide, combining the reporter-ion intensities between infected patient channels $(127 n, 128 n, 129 n, 130 n$, and $131 \mathrm{n})$ and non-infected patient channels $(126,127 \mathrm{c}$, 128c, 129c, and 130c). To test the ratio's accuracy and biological significance, technical and biological variability 
were calculated for each protein ratio. A ratio $p$ value and sample $p$ value were calculated for each variable. Furthermore, only proteins with a cut-off threshold value higher than 1.5 or lower than 0.67 were considered [29-31].

\section{SAA 1/2 PRM analysis}

Parallel reaction monitoring (PRM) analysis was performed on ten infected and ten non-infected plasma samples using a Q-Exactive Plus mass spectrometer (ThermoFisher), as previously described [32]. The aim was to verify the discovery results.

Each sample was loaded into a PepMap precolumn $(2 \mathrm{~cm} \times 75 \mu \mathrm{m}$ i.d., C18, $3 \mu \mathrm{m}$, and $100 \AA$ pore size $)$. Subsequent separation was performed in a PepMap col-

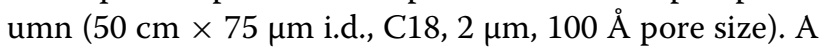
mixture of mobile $A$ and $B$ phases was used for peptide elution. The phase A solvent was composed of $0.1 \%(\mathrm{v} / \mathrm{v})$ formic acid (Biosolve) and HPLC-grade water (Romil); the phase B solvent was composed of $0.1 \%(\mathrm{v} / \mathrm{v})$ formic acid in HPLC-grade acetonitrile (Romil). To perform the separation, a linear gradient of $5-35 \%$ solvent $\mathrm{B}$ at $250 \mathrm{~nL} / \mathrm{min}$ for $60 \mathrm{~min}$ was set and it was followed by a washing step (35-90\% of solvent B for $10 \mathrm{~min}$ ).

Three masses were targeted (doubly and triply charged ions), corresponding to total SAA, but also specifically to SAA1 and SAA2. The selection of the different peptides was performed considering two different criteria: a previous SAA PRM study and the results of our quantitative proteomic analysis [32]. The three peptides selected in this way were tryptic peptides associated to each isoform.

This inclusion list triggered targeted scans at a resolving power of 70,000, with an isolation width of $1 \mathrm{Th}$ around the $\mathrm{m} / \mathrm{z}$ of interest, an AGC target of $1 \times 10^{6}$, a maximum injection time of $100 \mathrm{~ms}$, and a normalized collision energy of $27 \%$ in a higher-energy c-trap dissociation (HCD) cell.

Data analysis Data were analyzed using the targeted MS/MS feature available in Skyline v3.5 software [33]. In order to confirm the identity of the peptides, a data dependent acquisition spectral library of annotated reference MS/MS spectra was created from the two pools of plasma samples composed of infected and non-infected patients. Peptides were quantified by extracting the peak areas of accurate fragment ions $(<6 \mathrm{ppm})$, and they were then integrated across the peptides' elution profiles. For each peptide, transition peak areas were normalized by the average of the sum of the transition peak areas for all the peptides across the runs.

\section{SAA1/2 ELISA measurement}

The Vascular Injury Panel-I electrochemiluminescence $(\mathrm{ECL})$ assay was used to determine the levels of SAA1/2 in 40 stroke patients, as per the manufacturer's instructions (Meso Scale Discovery, Gaithersburg, MD). Each plasma sample was diluted 1:1000 with using sample diluent provided by the kit. An ECL detection system using multi-array technology (SECTOR Imager 2400, Meso Scale Discovery) was used to determine analyte concentrations. Samples were measured in a single detection.

\section{Statistical analyses}

Statistical analyses were carried out using SPSS software (v21, SPSS Inc., Chicago, IL). Analytes were not normally distributed, so the Mann-Whitney U-test was used to compare the two unpaired groups. Fisher's exact test and the Chi squared test were used to assess whether patients with and without infection were significantly different according to their gender, medical history, clinical data, laboratory values, lesion size, or TOAST. All statistical tests were two-tailed, and a $\mathrm{p}$ value $<0.05$ was considered statistically significant.

Multivariate analyses were performed to assess the associations between variables. The presence/absence of infection was set as the dependent variable, and SAA, CRP, WBC, and NIHSS were set as confounders. The model was validated using the bootstrap method. Categorical data were dichotomized according to the criteria in the table of demographic characteristics. Longitudinal data were also dichotomized according to the best cut-off obtained from area under the receiver operating characteristic (ROC) curve (AUC) analysis.

\section{Results}

Baseline population characteristics

Of 40 consecutively enrolled ischemic stroke patients, 21 developed an infection within 5 days of stroke onset (day 4 was the median day of infection development after the cerebrovascular event). Mean patient age was 79 years old (IQR: $70-82$ years) and 55\% of patients were men. Patients with severe strokes, resulting in higher NIHSS values at hospital admission, were more prone to developing an infection than patients with minor strokes. Other factors, such as hypertension, diabetes mellitus, or smoking, did not significantly affect the development of an infection. Nevertheless, according to the modified Rankin Scale, patient outcome appeared to be significantly affected by the development of an infection, as most of the patients with a poor outcome had developed an infection during their hospital stay.

At hospital admission, levels of WBC and CRP were within the normal range in both groups, with no significant differences found between infected and noninfected patients. Patients' demographic characteristics are summarized in Table 1. 
Table 1 Baseline data

\begin{tabular}{|c|c|c|c|c|}
\hline & $\begin{array}{l}\text { All patients } \\
(n=40)\end{array}$ & $\begin{array}{l}\text { No infection } \\
(n=19)\end{array}$ & $\begin{array}{l}\text { Infection } \\
(n=21)\end{array}$ & $p$ value \\
\hline \multicolumn{5}{|l|}{ Demographic data } \\
\hline Age, median (IQR) & $79.2(70.4-82)$ & $78.3(74-80.5)$ & $80.4(69.5-83)$ & 0.78 \\
\hline Female sex, $n(\%)$ & $18(45)$ & $9(47.4)$ & $9(42.9)$ & 1 \\
\hline \multicolumn{5}{|l|}{ Medical history, $n(\%)$} \\
\hline Hypertension & $31(77.5)$ & $12(63.2)$ & $19(90.5)$ & 0.06 \\
\hline Atrial fibrillation & $9(22.5)$ & $3(15.8)$ & $6(28.6)$ & 0.17 \\
\hline Current smoking & $11(27.5)$ & $5(26.3)$ & $6(28.6)$ & 0.64 \\
\hline Diabetes mellitus & $7(17.5)$ & $4(21.1)$ & $3(14.3)$ & 0.69 \\
\hline Coronary heart disease & $10(25)$ & $4(21.1)$ & $6(28.6)$ & 0.72 \\
\hline Previous stroke & $11(27.5)$ & $5(26.3)$ & $6(28.6)$ & 1 \\
\hline \multicolumn{5}{|l|}{ Clinical data, median (IQR) } \\
\hline NIHSS at admission & $5.5(2-12)$ & $3(2-7)$ & $12(4-14)$ & 0.01 \\
\hline \multicolumn{5}{|c|}{ Laboratory values, median (IQR) } \\
\hline WBC (g/l) & $8.6(6.8-10.1)$ & $7.7(6.2-9.3)$ & $9.3(7.4-11.2)$ & 0.14 \\
\hline $\operatorname{CRP}(\mathrm{mg} / \mathrm{l})$ & $3.6(3-9.1)$ & $3.6(3-6.8)$ & $4.8(3-17.4)$ & 0.22 \\
\hline \multicolumn{5}{|l|}{ Lesion size on MR, DWlb } \\
\hline Small $\left(1-10 \mathrm{~mm}^{3}\right)$ & $23(27.5)$ & $13(68.4)$ & $10(47.6)$ & 0.47 \\
\hline Medium $\left(10-100 \mathrm{~mm}^{3}\right)$ & $8(20)$ & $3(15.8)$ & $5(23.8)$ & 0.75 \\
\hline Large $\left(>100 \mathrm{~mm}^{3}\right)$ & $1(2.5)$ & $0(0)$ & $1(4.8)$ & 0.69 \\
\hline \multicolumn{5}{|l|}{ TOAST } \\
\hline Large vessel stroke & $8(20)$ & $3(15.8)$ & & 0.69 \\
\hline Cardioembolic stroke & $8(20)$ & $4(21.1)$ & & 1 \\
\hline Microangipathic stroke & $14(35)$ & $5(26.3)$ & & 0.33 \\
\hline Other & $0(0)$ & $0(0)$ & & \\
\hline Unknown & $10(25)$ & $7(36.8)$ & & 0.15 \\
\hline
\end{tabular}

\section{Proteomic results}

In order to find a biomarker able to distinguish, at hospital admission, which patients will and will not develop a post-stroke infection, the proteomes of five infected and five non-infected stroke patients were compared using quantitative proteomic analysis. Applying the criteria of a maximum of $1 \%$ FDR and at least two unique peptides, 266 proteins were quantified (Additional file 1: Table 1). Of all the proteins, serum amyloid A1 appeared to be the only significantly $(p=0.007)$ regulated protein between the two groups of patients, with a ratio of 2.2 after Bonferroni correction.

To verify the results obtained by the $\mathrm{TMT}^{10}$ plex during the discovery phase, a further PRM analysis was performed on a new batch of patients. Consequently, we targeted three transitions of the tryptic SFFSFLGEAFDGAR peptide in 10 infected and 10 non-infected patients. This peptide is common to all the different isoforms of acute-phase SAA. By measuring its concentration, therefore, we were sure to measure the total amount SAA present in blood and not only that of one of the different described isoforms. As shown in Fig. 1, the concentration of SFFSFLGEAFDGAR was significantly higher $(\mathrm{p}<0.001)$ in infected patients than in noninfected ones, confirming that there was a clear overproduction of SAA in patients who went on to develop an infection.

\section{Different SAA isoforms for infection development}

Further PRM analyses were performed on the same 20 patients in order to evaluate whether either of the acute phase isoforms (SAA1 and SAA2) had a more significant effect on infection and inflammatory processes. The high sequence-similarity between the SAA1 and SAA2 isoforms prevented an evaluation of their effects using classic ELISAs. The present study measured three transitions in the FFGHGAEDSLADQAANEWGR peptide (unique to SAA1) and GPGGAWAAEVISNAR peptide (unique to SAA2) across 10 infected and 10 non-infected patients. As Additional file 2: Fig. 1 shows, both peptides were significantly $(\mathrm{p}<0.001)$ more abundant in infected patients than in non-infected ones. 


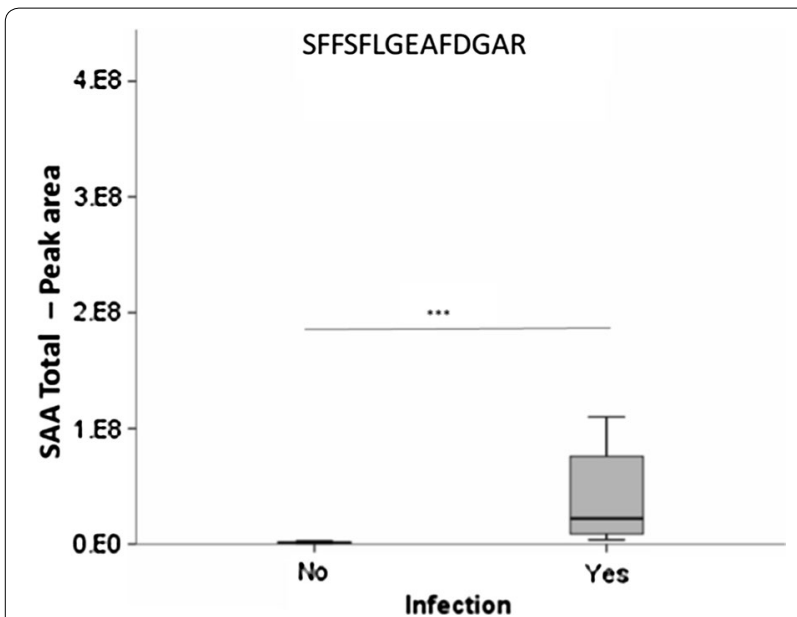

Fig. 1 Ratio of SFFSFLGEAFDGAR peptide abundance between patients who went on to develop an infection and those who did not. The SFFSFLGEAFDGAR peptide is common to all the different isoforms of SAA

\section{Kinetics of serum amyloid A1/2}

Serum amyloid A1/2 plasma concentrations were subsequently measured in a new group of 21 infected and 19 non-infected patients in order to validate the previous proteomic results. Concentrations of this acute-phase reactant molecule were measured at hospital admission and at 1,3 , and 5 days after hospitalization, using an SAA1/2 ELISA assay. Initially, analyses were performed separately in those patients used for the discovery step and in those used for the verification/validation step. As Additional file 3: Fig. 2 shows, in both cases, SAA concentrations were significantly higher in patients who went on to develop a post-stroke infection than in those who did not. Subsequently, analyses were performed again when all the patients were evaluated together. As Fig. 2 shows, peptide concentrations were again significantly higher in infected patients than in non-infected patients, at all time points, particularly at 3 days $(p=0.01)$ and 5 days $(p=0.01)$ after stroke onset.

SAA measurements were evaluated to distinguish between the two groups of patients at D0, D1, D3, and D5. As Table 2 shows, the accuracy of SAA measurements in distinguishing which patients went on to develop an infection and which did not reached values of $73.2 \%$ (cut-off: $14.2 \mu \mathrm{g} / \mathrm{mL}$ ) and 77.1\% (cut-off: $8.8 \mu \mathrm{g} / \mathrm{mL}$ ) at hospital admission and 1 day after, respectively. Three days after hospitalization, the AUC of SAA was slightly better, reaching a value of $80.7 \%$ (cut-off: $21.4 \mu \mathrm{g} / \mathrm{mL}$ ), and 5 days after hospitalization, the AUC was 76.7\% (cut-off: $87.7 \mu \mathrm{g} / \mathrm{mL}$ ).

To evaluate the capacity of SAA $1 / 2$ measurement to rule-in patients at risk of infection, we set specificity (SP) at between 90 and $100 \%$. At hospital admission, with a 94.7\% SP, SAA measurement reached $42.9 \%$ sensitivity

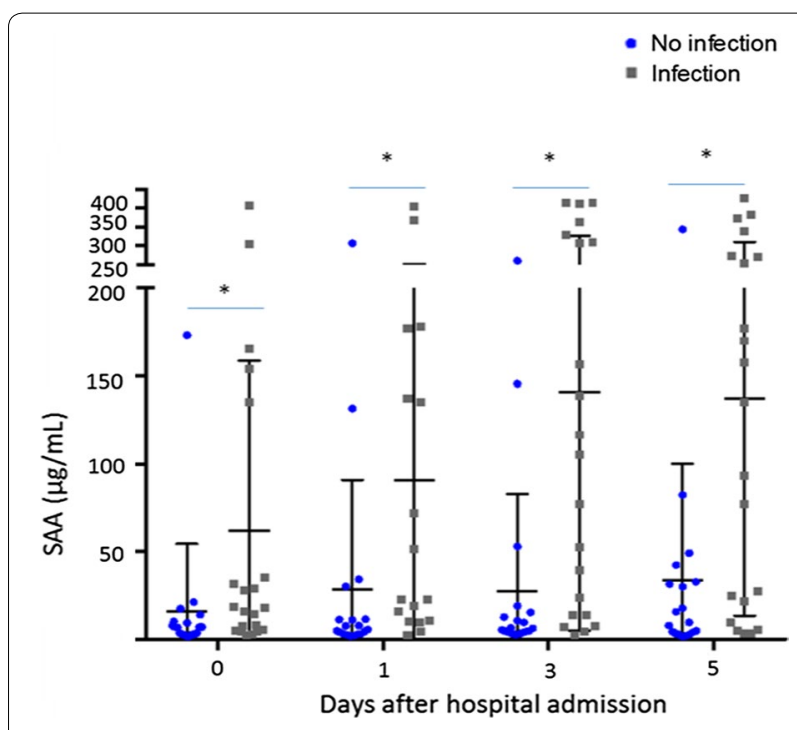

Fig. 2 Kinetics of SAA concentrations at hospital admission, 1, 3, and 5 days after stroke onset. Text under Fig. 2 in PDF: SAA concentrations are shown by grey square for infected patients and by blue square for non-infected patients. Comparisons between the two groups were made using the Mann-Whitney $U$ test. Asterisk significance level reported after the Bonferroni correction $(p<0.05)$

(SE) and a partial AUC of 2.5\% (Table 2). Three days after hospitalization, SP reached $100 \%$, SE was $33.3 \%$, and the partial AUC was $3.6 \%$ (Table 2). All the AUC and pAUC curves obtained at the different time points are represented in Fig. 3. These AUC and pAUC values were obtained using different cut-off concentrations corresponding to the best combination of SP and SE.

However, due to the high variability of the SAA concentrations obtained, we decided to evaluate the possibility of using a ratio based on those concentrations to predict the development of an infection. As Table 2 shows, patients who went on to develop an infection during their hospital stay, presented with an average 2.4 times greater concentration of SAA on D3 than on D1. For patients who did not become infected, average SAA concentrations remained very similar (ratio of 0.97 ), with no significant increase, thus suggesting that this ratio could be used as an indicator of patients at risk.

\section{Multivariate analyses}

Finally, we performed multivariate analyses in order to confirm that SAA was a promising biomarker of poststroke infection and to assess whether it was an independent predictive factor. The presence of infection was set as the dependent variable, and the significantly regulated parameters according to the patients' demographic characteristics (NIHSS and SAA) were set as confounders. WBC and CRP were also included in the confounder group because they are widely used in clinical practice. 
Table 2 Capacity of plasma concentrations of SAA to distinguish between patients who went on to develop an infection and those who did not

\begin{tabular}{|c|c|c|c|c|c|c|c|c|c|}
\hline \multirow[t]{3}{*}{ Day } & \multicolumn{2}{|c|}{ Number of patients } & \multicolumn{3}{|c|}{$\begin{array}{l}\text { Mean SAA concentration } \\
(\mu \mathrm{g} / \mathrm{mL}) \pm \text { SD }\end{array}$} & \multicolumn{4}{|l|}{ ROC curve } \\
\hline & No infection & Infection & No infection & Infection & $p$ value & AUC (95\% Cl) & Cut-off & $\mathrm{SP} \%(95 \% \mathrm{CI})$ & SE\% (95\% Cl) \\
\hline & & & & & & pAUC $(95 \% \mathrm{Cl})$ & & SP $90-100 \%(95 \% \mathrm{Cl})$ & \\
\hline \multirow[t]{2}{*}{0} & 19 & 21 & $16 \pm 38.4$ & $61.8 \pm 96.7$ & 0.01 & $73.2(55.9-87)$ & 14.2 & $84.2(68.4-100)$ & $61.9(38.1-81)$ \\
\hline & & & & & & $2.53(0-6.7)$ & 24.7 & $94.7(84.2-100)$ & $42.9(23.8-62.02)$ \\
\hline \multirow[t]{2}{*}{1} & 19 & 17 & $28.2 \pm 62.6$ & $57.7 \pm 92.9$ & 0.005 & $77.1(60.1-92)$ & 8.8 & $63.2(42.1-84.2)$ & $88.2(70.6-100)$ \\
\hline & & & & & & $2.3(0.6-6.5)$ & 133.4 & $94.7(84.2-100)$ & $35.3(11.8-58.8)$ \\
\hline \multirow[t]{2}{*}{3} & 19 & 21 & $27.6 \pm 55.2$ & $140.9 \pm 136$ & 0.001 & $80.7(66.2-93.2)$ & 21.4 & $84.2(68.4-100)$ & $71.4(52.4-90.5)$ \\
\hline & & & & & & $3.6(1.7-7.6)$ & 233.9 & $100(100-100)$ & $33.3(14.3-52.4)$ \\
\hline \multirow[t]{2}{*}{5} & 19 & 21 & $33.6 \pm 66.4$ & $137 \pm 123.5$ & 0.003 & $76.7(61.2-89.7)$ & 87.8 & $94.7(84.2-100)$ & $57.1(38.1-76.2)$ \\
\hline & & & & & & $3.5(0.5-7.6)$ & 87.8 & $94.7(84.2-100)$ & $57.1(38.1-76.2)$ \\
\hline
\end{tabular}

As Table 3 shows, SAA was the only marker that displayed a relationship with the development of post-stroke infections, thus confirming and validating the possibility of measuring SAA concentrations as a biomarker of infection in stroke patients.

\section{Discussion}

The present study highlighted the capacity of proteomics to identify protein biomarkers that could assist in the detection of stroke patients at a high risk of developing post-stroke infection [34]. Using isobaric labeling methods, we first compared the plasma samples of five infected stroke patients and five non-infected stroke patients. We found that concentrations of serum amyloid A1 were overexpressed in patients who went on to develop an infection. This first approach was then verified using parallel reaction monitoring in 20 stroke patients (10 infected and 10 non-infected). Finally, the SAA1/2 concentrations of 40 ischemic stroke patients were confirmed using ELISA kits. The results demonstrated that
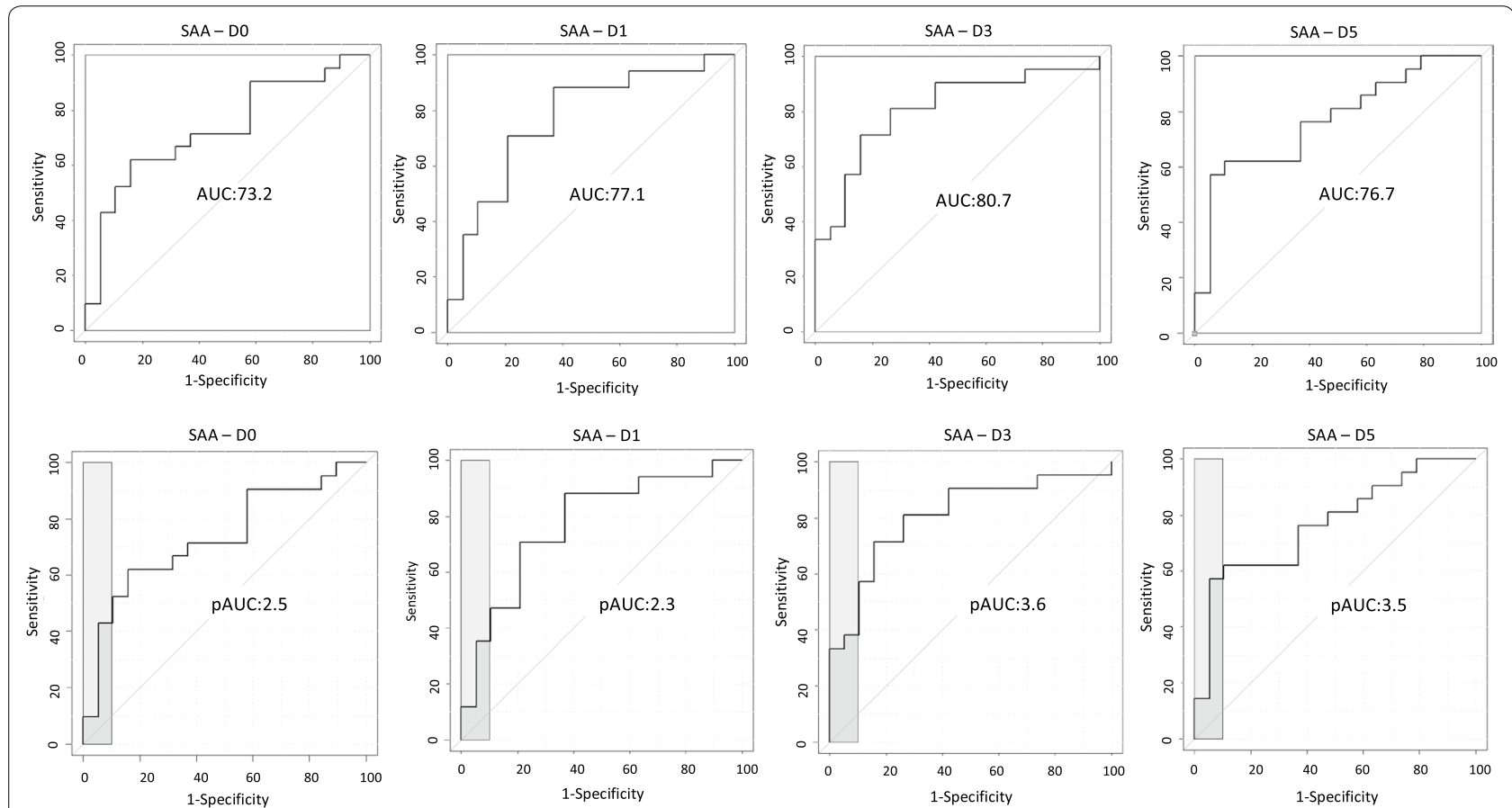

Fig. 3 ROC curves for SAA representing the ability to differentiate between patients who went on to develop an infection and those who did not on the day of hospital admission (D0) and 1 day (D1), 3 days (D3), and 5 days after (D5) stroke onset 
Table 3 Multivariate analyses of different factors predicting the presence of infection

\begin{tabular}{llll}
\hline Infection & & & \\
\hline Predictors & OR & $\mathbf{9 5 \%} \mathbf{C l}$ & $\boldsymbol{p}$ \\
\hline SAA & 3.68 & $(1.27-10.6)$ & 0.047 \\
CRP & 0.37 & $(0.12-1.17)$ & 0.09 \\
WBC & 1.24 & $(0.86-1.79)$ & 0.24 \\
NIHSS & 1.18 & $(1.01-1.38)$ & 0.031 \\
\hline
\end{tabular}

SAA1/2 is an efficient infection-risk prediction marker in stroke patients.

SAA1/2 has already been described as a potential marker of inflammation and infection in several pathological conditions, including stroke and subarachnoid hemorrhage, [35]. In a case-controlled study involving 54 patients, levels of acute-phase proteins were significantly higher in stroke patients who developed an infection during the month preceding a cerebrovascular event than in those who did not develop one. During the month following the stroke, concentrations of SAA started being significantly higher in patients who went on to develop an infection than in those who had only had an infection preceding the cerebrovascular event at 3 days after the onset of symptoms [36]. This highlighted that the acute-phase response was clearly related to the development of infections. In another study, of 60 patients, levels of SAA were significantly higher in 45 patients with stroke than in the 15 control patients without stroke. SAA concentrations increased between days one and three in patients with a cerebral infarction complicated by an infectious inflammatory process [37]. These results suggested a correlation between the acute-phase response and the development of an infection. Nevertheless, to the best of our knowledge, until now no one had evaluated the ability of these acute-phase molecules to act as predictors of infection.

In a population of 81 subarachnoid hemorrhage patients, SAA concentrations measured at hospital admission predicted which patients would develop an infection during their hospital stay with an accuracy of $76 \%$ [38]. We therefore decided to perform the same analysis using ischemic stroke patients in the present study. As already shown, very similar results were obtained.

To the best of our knowledge, our study is the first to assess the predictive value of SAA concentrations while taking into account the time points of measurements as well as the diagnosis. We found that the SAA concentration was able to detect $42.9 \%$ of the stroke patients who had a very high certainty of going on to develop an infectious complication.

Human SAA is an acute-phase protein primarily expressed by the liver [39]. There are four different but closely related genes responsible of the protein's different isoforms. In humans, the production of SAA1 and SAA2 takes place under inflammatory conditions. SAA3 is a pseudo-gene, and SAA4 encodes a protein that is produced constitutively [40]. Inflammatory SAA1 and SAA2 share around $90 \%$ of their gene sequence. Due to the similarities between both, immunoassays have been unable to differentiate between them [39], and studies to date have been unable to determine which of the isoforms is most associated with infectious and inflammatory processes. In the present study, we used the PRM method to track each isoform and evaluate its contribution. As shown in Additional file 2: Fig. 1, both SAA1 and SAA2 are related to infection development. The FFGHGAEDSLADQAANEWGR peptide, unique to SAA1, and the tryptic SAA2 GPGGAWAAEVISNAR peptide appeared to be significantly more abundant in patients suffering from an infection than in patients without one.

SAA concentrations are most likely higher in stroke patients suffering from infections due to its role in attracting leukocytes and immune cells to the sites of tissue damage, infection, or inflammation [41, 42]. As previously described, inflammation is an important part of the reactions taking place after an ischemic event. Indeed, blood derived leukocytes and microglia will be activated from minutes to hours after a cerebrovascular event [43]. Recruitment, activation, and adhesion of leukocytes to the endothelium will happen at the same time as neutrophils and monocytes/macrophages transmigrate into the location of the cerebral infarction [44]. During this process of brain damage, the acute-phase response will also activate acute-phase proteins as SAA, CRP, haptoglobin, $\alpha 1$-acid glycoprotein, $\alpha 1$-antichymiotripsin appear increasingly in the blood [44].

The present study has certain limitations. (1) The cohort was small and its results should be validated in a larger cohort of patients in order to have sufficient samples for the subgroup analyses (infection, no-infection). (2) The study proposed SAA as a promising prognostic infection marker in stroke patients. Nevertheless, combining SAA concentrations with other clinical scales (NIHSS) or scores could improve the accuracy of the association. Different combinations should be tested to evaluate the potential added value of a panel of markers. (3) Another point which remains to be investigated is why SAA concentrations become elevated in patients developing an infection much earlier than CRP does, for example. The present study postulated that this was due to its role in inflammation, but are we thus measuring inflammation or are we facing a post-infection inflammation phenomenon? As previously reported, the acute-phase response is more prominent in patients who develop an infection during hospitalization, but 
SAA concentrations were already higher when a previous bacterial infection was present. A detailed study should be performed to compare all these factors. (4) Different isoforms of SAA did not seem to act differently in conditions of inflammation. Nevertheless, the present study was only able to measure one tryptic peptide from each isoform. Further studies should target different peptides corresponding to the different isotypes of each isoform in order to perform a give a more detailed analysis of the role of SAA in infected stroke patients. Finally, to translate this study's results into clinical practice, a point of care test should be developed in order to provide results in minutes and ensure better, faster patient management.

\section{Conclusions}

In a small cohort of stroke patients, we were able to demonstrate that the concentrations of SAA1/2 measured at hospital admission could be used to predict post-stroke infection. Applying SAA measurement in clinical settings could drastically improve patient management and, consequently, their associated outcomes. Further large, multicenter validation studies are needed to confirm these results.

\section{Additional files}

Additional file 1. List of proteins identified when comparing the proteomes of five infected and five non-infected patients.

Additional file 2. Ratio of FFGHGAEDSLADQAANEWGR and GPGGAWAAEVISNAR peptide abundance between patients who went on to develop an infection and those who did not.

Additional file 3. Kinetics of SAA concentrations at hospital admission, 1 day, 3 days and 5 days after stroke onset, including discovery step patients only (a) and verification step patients only (b).

\begin{abstract}
Abbreviations
AUC: area under the ROC curve; A2DS2: age, atrial fibrillation, dysphagia, sex, stroke severity; CDC: Centers for Disease Control and Prevention; Cl: confidence interval; CID: collision-induced dissociation; CRP: C-reactive protein; CSF: cerebrospinal fluid; CT: computed tomography; ELISA: enzyme-linked immunosorbent assay; ECG: electrocardiogram; FDR: false discovery rate; HCD: higher-energy collisional dissociation; HPLC: high-performance liquid chromatography; HRP: horseradish peroxidase; IQR: interquartile range; IPG: immobilized pH gradient; MSD: mesoscale discovery; NIHSS: National Institutes of Health Stroke Scale; OGE: off-gel electrophoresis; OT: OrbiTrap; PRM: parallel reaction monitoring; ROC: receiver operating characteristic; $P C T$ : procalcitonin; SAA1/2: serum amyloid 1/2; SE: sensitivity; SP: specificity; TEAB: tetraethylammonium bromide; TCEP: tris-(2-carboxyethil) phosphine hydrochloride; TMT: tandem mass tag; TOAST: trial of org 1072 in acute stroke treatment; WBC: white blood cells.
\end{abstract}

\section{Authors' contributions}

LA contributed to data acquisition and analysis as well as to drafting the manuscript. VLF contributed to data acquisition and analysis as well as to drafting the manuscript. JS contributed to sample collection and to correction and final approval of the manuscript. JM contributed to sample collection and to correction and final approval of the manuscript. MK contributed to sample collection and to correction and final approval of the manuscript. JCS contributed to data interpretation and to correction. All authors read and approved the final manuscript.

\author{
Author details \\ ${ }^{1}$ Translational Biomarker Group, Department of Human Protein Sciences, \\ University of Geneva, Rue Michel Servet 1, 1211 Geneve 4, Switzerland. \\ 2 Department of Neurology, University Hospital of Zurich, Zurich, Switzerland. \\ ${ }^{3}$ Department of Medicine, Universitat Autònoma de Barcelona, Barcelona, \\ Spain.
}

\section{Acknowledgements}

We wish to thank all the patients, nurses, and physicians who participated this study

\section{Competing interests}

The authors declare that they have no competing interests.

\section{Availability of data and materials}

All the datasets included in the present manuscript are presented in the main manuscript or additional supporting files.

\section{Ethics approval and consent to participate}

We performed a post hoc analysis of a prospective cohort study including 40 ischemic stroke patients (ClinicalTrials.gov.NCT00390962) who were hospitalized consecutively at the University Hospital of Basel (Switzerland) between November 2006 and November 2007. The study was conducted according to the principles expressed in the Declaration of Helsinki, and the study protocol was approved by the local ethics committee. Before enrolment, informed consent was obtained from patients, their relatives, or their legal guardians.

\section{Publisher's Note}

Springer Nature remains neutral with regard to jurisdictional claims in published maps and institutional affiliations.

Received: 1 March 2017 Accepted: 3 July 2017

Published online: 12 July 2017

\section{References}

1. GBD 2013 Mortality and Causes of Death Collaborators. Global, regional, and national age-sex specific all-cause and cause-specific mortality for 240 causes of death, 1990-2013: a systematic analysis for the Global Burden of Disease Study 2013. Lancet 2015;385(9963):117-71.

2. Truelsen T, Piechowski-Jozwiak B, Bonita R, Mathers C, Bogousslavsky J, Boysen G. Stroke incidence and prevalence in Europe: a review of available data. Eur J Neurol. 2006;13:581-98.

3. Warlow C, Sudlow C, Dennis M, Wardlaw J, Sandercock P. Stroke. Lancet. 2003:362:1211-24.

4. Langhorne P, Stott DJ, Robertson L, MacDonald J, Jones L, McAlpine C, Dick F, Taylor GS, Murray G. Medical complications after stroke. Multicent Study. 2000;31:1223-9.

5. Tirschwell DL, Kukull WA, Longstreth WT Jr. Medical complications of ischemic stroke and length of hospital stay: experience in Seattle, Washington. J Stroke Cerebrovasc Dis. 1999;8:336-43.

6. Al-Khaled M, Matthis C, Eggers J. Predictors of in-hospital mortality and the risk of symptomatic intracerebral hemorrhage after thrombolytic therapy with recombinant tissue plasminogen activator in acute ischemic stroke. J Stroke Cerebrovasc Dis. 2014;23:7-11.

7. Hug A, Murle B, Dalpke A, Zorn M, Liesz A, Veltkamp R. Usefulness of serum procalcitonin levels for the early diagnosis of stroke-associated respiratory tract infections. Neurocrit Care. 2011;14:416-22.

8. Chamorro Á, Urra X, Planas AM. Infection after acute ischemic stroke. Manif Brain Induc Immunodepress. 2007;38:1097-103.

9. Weimar C, Roth MP, Zillessen G, Glahn J, Wimmer ML, Busse O, Haberl RL, Diener HC. Complications following acute ischemic stroke. Eur Neurol. 2002:48:133-40.

10. Westendorp WF, Nederkoorn PJ, Vermeij JD, Dijkgraaf MG, van de Beek D. Post-stroke infection: a systematic review and meta-analysis. BMC Neurol. 2011;11:110 
11. Marrie TJ, Wu L. Factors influencing in-hospital mortality in communityacquired pneumonia: a prospective study of patients not initially admitted to the ICU. Chest. 2005;127:1260-70.

12. Chamorro A, Urra X, Planas AM. Infection after acute ischemic stroke: a manifestation of brain-induced immunodepression. Stroke. 2007;38(3):1097-103.

13. Dirnagl U, Klehmet J, Braun JS, Harms H, Meisel C, Ziemssen T, Prass K, Meisel A. Stroke-induced immunodepression: experimental evidence and clinical relevance. Stroke. 2007;38:770-3.

14. Azurmendi L, Degos V, Tiberti N, Kapandji N, Sanchez-Pena P, Sarrafzadeh A, Puybasset L, Turck N, Sanchez JC. Neopterin plasma concentrations in patients with aneurysmal subarachnoid hemorrhage: correlation with infection and long-term outcome. J Neurosurg. 2016;124(5):1287-99.

15. Fluri F, Morgenthaler NG, Mueller B, Christ-Crain M, Katan M. Copeptin, procalcitonin and routine inflammatory markers-predictors of infection after stroke. PLoS ONE. 2012;7:e48309.

16. Gumbinger C, Hug A, Murle B, Berger B, Zorn M, Becker KP, Zimmermann $\mathrm{S}$, Dalpke AH, Veltkamp R. Early blood-based microbiological testing is ineffective in severe stroke patients. J Neurol Sci. 2013;325:46-50.

17. Hoffmann S, Malzahn U, Harms H, Koennecke HC, Berger K, Kalic M, Walter G, Meisel A, Heuschmann PU. Development of a clinical score (A2DS2) to predict pneumonia in acute ischemic stroke. Stroke. 2012:43:2617-23.

18. Smith CJ, Bray BD, Hoffman A, Meisel A, Heuschmann PU, Wolfe CD, Tyrrell PJ, Rudd AG. Can a novel clinical risk score improve pneumonia prediction in acute stroke care? A UK multicenter cohort study. J Am Heart Assoc. 2015:4:e001307.

19. Ji R, Shen H, Pan Y, Wang P, Liu G, Wang Y, Li H, Wang Y. Novel risk score to predict pneumonia after acute ischemic stroke. Stroke. 2013:44:1303-9.

20. Finlayson O, Kapral M, Hall R, Asllani E, Selchen D, Saposnik G. Risk factors, inpatient care, and outcomes of pneumonia after ischemic stroke. Neurology. 2011;77:1338-45.

21. Garner JS, Jarvis WR, Emori TG, Horan TC, Hughes JM. CDC definitions for nosocomial infections. Am J Infect Control. 1988;16:128-40.

22. Adams HP, Bendixen BH, Kappelle LJ, Biller J, Love BB, Gordon DL. Classification of subtype of acute ischemic stroke. Definitions for use in a multicenter clinical trial. TOAST. Trial of Org 10172 in acute stroke treatment. Stroke. 1993:24(1):35-41.

23. Dayon L, Turck N, Kienle S, Schulz-Knappe P, Hochstrasser DF, Scherl A, Sanchez JC. Isobaric tagging-based selection and quantitation of cerebrospinal fluid tryptic peptides with reporter calibration curves. Anal Chem. 2010;82:848-58.

24. Dayon L, Turck N, Scherl A, Hochstrasser DF, Burkhard PR, Sanchez $J$-C. From relative to absolute quantification of tryptic peptides with tandem mass tags: application to cerebrospinal fluid. CHIMIA Int J Chem. 2010;64:132-5.

25. Dayon L, Pasquarello C, Hoogland C, Sanchez J-C, Scherl A. Combining low- and high-energy tandem mass spectra for optimized peptide quantification with isobaric tags. J Proteomics. 2010;73:769-77.

26. Gluck F, Hoogland C, Antinori P, Robin X, Nikitin F, Zufferey A, Pasquarello C, Fétaud V, Dayon L, Müller M, et al. EasyProt-an easy-to-use graphical platform for proteomics data analysis. J Proteomics. 2013;79:146-60.

27. Elias JE, Gygi SP. Target-decoy search strategy for increased confidence in large-scale protein identifications by mass spectrometry. Nat Methods. 2007:4:207-14.

28. Breitwieser FP, Muller A, Dayon L, Kocher T, Hainard A, Pichler P, SchmidtErfurth U, Superti-Furga G, Sanchez JC, Mechtler K, et al. General statistical modeling of data from protein relative expression isobaric tags. J Proteome Res. 2011;10:2758-66.
29. Tiberti N, Hainard A, Lejon V, Robin X, Ngoyi DM, Turck N, Matovu E, Enyaru J, Ndung'u JM, Scherl A, et al. Discovery and verification of osteopontin and beta-2-microglobulin as promising markers for staging human African trypanosomiasis. Mol Cell Proteomics MCP. 2010;9:2783-95.

30. Tan HT, Tan S, Lin Q, Lim TK, Hew CL, Chung MC. Quantitative and temporal proteome analysis of butyrate-treated colorectal cancer cells. Mol Cell Proteomics. 2008;7:1174-85.

31. Salvisberg C, Tajouri N, Hainard A, Burkhard PR, Lalive PH, Turck N. Exploring the human tear fluid: discovery of new biomarkers in multiple sclerosis. Proteomics Clin Appl. 2014;8:185-94.

32. Kim YJ, Gallien S, El-Khoury V, Goswami P, Sertamo K, Schlesser M, Berchem G, Domon B. Quantification of SAA1 and SAA2 in lung cancer plasma using the isotype-specific PRM assays. Proteomics. 2015:15:3116-25.

33. MacLean B, Tomazela DM, Shulman N, Chambers M, Finney GL, Frewen B, Kern R, Tabb DL, Liebler DC, MacCoss MJ. Skyline: an open source document editor for creating and analyzing targeted proteomics experiments. Bioinformatics. 2010;26:966-8.

34. Garcia-Berrocoso T, Penalba A, Boada C, Giralt D, Cuadrado E, Colome N, Dayon L, Canals F, Sanchez JC, Rosell A, Montaner J. From brain to blood: new biomarkers for ischemic stroke prognosis. J Proteomics. 2013:94:138-48.

35. Azurmendi L, Degos V, Tiberti N, Kapandji N, Sanchez P, Sarrafzadeh A Puybasset L, Turck N, Sanchez J-C. Measuring serum amyloid A for infection prediction in aneurysmal subarachnoid hemorrhage. J Proteome Res. 2015;14:3948-56.

36. Syrjanen J, Teppo AM, Valtonen VV, livanainen M, Maury CP. Acute phase response in cerebral infarction. J Clin Pathol. 1989;42:63-8.

37. Ilzecka J, Stelmasiak Z. Prognostic importance of monitoring serum amyloid A protein (SAA) in patients with cerebral infarction. Acta Clin Croat. 2000;39:139-46.

38. Azurmendi L, Degos V, Tiberti N, Kapandji N, Sanchez P, Sarrafzadeh A Puybasset L, Turck N, Sanchez JC. Measuring serum amyloid A for infection prediction in aneurysmal subarachnoid hemorrhage. J Proteome Res. 2015;14:3948-56.

39. Uhlar CM, Whitehead AS. serum amyloid A, the major vertebrate acutephase reactant. Eur J Biochem. 1999;265:501-23.

40. Malle EDBF. Human serum amyloid A (SAA) protein: a prominent acutephase reactant for clinical practice. Eur J Clin Invest. 1996;26:427-35.

41. Gabay C, Kushner I. Acute-phase proteins and other systemic responses to inflammation. N Engl J Med. 1999:340:448-54.

42. Nakayama T, Sonoda S, Urano T, Yamada T, Okada M. Monitoring both serum amyloid protein $\mathrm{A}$ and $\mathrm{C}$-reactive protein as inflammatory markers in infectious diseases. Clin Chem. 1993;39:293-7.

43. del Zoppo GJ, Becker KJ, Hallenbeck JM. Inflammation after stroke: Is it harmful? Arch Neurol. 2001;58:669-72.

44. Ahmad M, Graham SH. Inflammation after stroke: mechanisms and therapeutic approaches. Transl Stroke Res. 2010;1:74-84.

\section{Submit your next manuscript to BioMed Central and we will help you at every step:}

- We accept pre-submission inquiries

- Our selector tool helps you to find the most relevant journal

- We provide round the clock customer support

- Convenient online submission

- Thorough peer review

- Inclusion in PubMed and all major indexing services

- Maximum visibility for your research

Submit your manuscript at www.biomedcentral.com/submit

C BioMed Central 